\title{
Development of diabetes mellitus in aging transgenic mice following suppression of pancreatic homeoprotein IDX-1
}

\author{
Melissa K. Thomas, Octavia N. Devon, Jee H. Lee, Andreas Peter, David A. Schlosser, \\ Matthew S. Tenser, and Joel F. Habener \\ Laboratory of Molecular Endocrinology, Massachusetts General Hospital, Howard Hughes Medical Institute, \\ Harvard Medical School, Boston, Massachusetts, USA \\ Address correspondence to: Joel F. Habener, Laboratory of Molecular Endocrinology, Massachusetts General Hospital, \\ 55 Fruit Street, WEL320, Boston, Massachusetts 02114, USA. \\ Phone: (617) 726-5190; Fax: (617) 726-6954; E-mail: jhabener@partners.org.
}

Received for publication December 15, 2000, and accepted in revised form June 4, 2001.

\begin{abstract}
Monogenic forms of diabetes can result from mutations in genes encoding transcription factors. Mutations in the homeodomain transcription factor IDX-1, a critical regulator of pancreas development and insulin gene transcription, confer a strong predisposition to the development of diabetes mellitus in humans. To investigate the role of IDX-1 expression in the pathogenesis of diabetes, we developed a model for the inducible impairment of IDX-1 expression in pancreatic $\beta$ cells in vivo by engineering an antisense ribozyme specific for mouse IDX-1 mRNA under control of the reverse tetracycline transactivator (rtTA). Doxycycline-induced impairment of IDX-1 expression reduced activation of the Insulin promoter but activated the $I d x-1$ promoter, suggesting that pancreatic $\beta$ cells regulate IDX-1 transcription to maintain IDX-1 levels within a narrow range. In transgenic mice that express both rtTA and the antisense ribozyme construct, impaired IDX-1 expression elevated glycated hemoglobin levels, diminished glucose tolerance, and decreased insulin/glucose ratios. Metabolic phenotypes induced by IDX-1 deficiency were observed predominantly in male mice over 18 months of age, suggesting that cellular mechanisms to protect IDX-1 levels in pancreatic $\beta$ cells decline with aging. We propose that even in the absence of $I d x-1$ gene mutations, pathophysiological processes that decrease IDX-1 levels are likely to impair glucose tolerance. Therapeutic strategies to attain normal glucose homeostasis by restoring normal IDX-1 levels may be of particular importance for older individuals with diabetes mellitus.
\end{abstract}

J. Clin. Invest. 108:319-329 (2001). DOI:10.1172/JCI20012029.

\section{Introduction}

The pancreatic homeodomain transcription factor islet duodenum homeobox-1 (IDX-1), also known as IPF-1 (1), STF-1 (2), PDX-1 (3), and GSF (4), is an important regulator of normal glucose homeostasis (reviewed in refs. 5,6$)$. Heterozygosity for an inactivating mutation in IDX-1 is associated with autosomal dominant earlyonset diabetes (MODY 4; ref. 7) and heterozygosity for missense mutations in IDX-1 confers a predisposition to late-onset type 2 diabetes mellitus in humans $(8,9)$. In mouse models, heterozygous inactivation of the $I d x$ 1 gene results in impaired glucose tolerance (10), and pancreatic $\beta$ cell-specific inactivation of the $I d x-1$ gene leads to the development of diabetes (11).

IDX-1 is critical for the development of the pancreas. Homozygosity for an inactivating mutation in IDX-1 results in pancreatic agenesis in a child and $I d x-1$-null mice also have pancreatic agenesis $(3,12,13)$. IDX-1 transactivates several genes essential for the differentiated $\beta$ cell phenotype important for glucose sensing and metabolism in the $\beta$ cell, including insulin, glucose-transporter-2, and glucokinase (14-17).

The expression of IDX-1 is impaired by pathophysiological processes in the absence of specific gene muta- tions. For example, glucotoxicity, excess glucocorticoids, and excess lipids decrease IDX-1 expression in experimental model systems (18-23). Here we report the generation of a model in which IDX-1 expression is inducibly impaired in pancreatic $\beta$ cells in the setting of an intact $I d x-1$ gene. We identify an autoregulatory loop within pancreatic $\beta$ cells in which the $I d x-1$ promoter senses and compensates for IDX-1 deficiency, thereby maintaining IDX-1 levels within a limited range. Our studies indicate that reduction of IDX-1 levels in $\beta$ cells in conjunction with aging leads to metabolic dysfunction and suggest that restoration of normal IDX-1 function may be of particular clinical relevance in type 2 diabetes.

\section{Methods}

Plasmid and transgene construction. The PCMV-IDX-1 expression plasmid, encoding the cDNA for rat IDX-1; the -4.6-kb pIDX-1-pGL3 plasmid, encoding $-4.6 \mathrm{~kb}$ of the mouse IDX-1 promoter and a luciferase reporter; and the pcDNA3-E12 and pcDNA3-E47 expression plasmids have been described previously (24-26). FarFlat-CAT, encoding pentamerized glucose-responsive elements Far and Flat derived from the rat insulin I promoter, was a gift from L.G. Moss 
(New England Medical Center and Tufts University School of Medicine, Boston, Massachusetts, USA). The tet operator-luciferase reporter plasmid pUHC 13-3 (27), the tet operator-multiple cloning site plasmid pUHD 10-3 (28), and the PCMV-rtTA plasmid pUHG 17-1 (29) were obtained from H. Bujard (Zentrum für Molekulare Biologie der Universitaet Heidelberg, Heidelberg, Germany).

To construct the RIP-rtTA transgene plasmid, a rat insulin II promoter fragment spanning -660 to +16 was first subcloned into the vector pSP72 (Promega Corp., Madison, Wisconsin, USA). A XhoI/EcoRI fragment of RIP-pSP72 encoding the RIP sequences was excised from PSP72 and cloned into XhoI/EcoRI sites within the rtTA plasmid PUHG 17-1 from which human CMV promoter sequences had been removed. The junctions of the RIP-rtTA plasmid were verified by automated sequencing. The Tet-ASRZ-IDX-1 transgene plasmid was created by first generating a doublestranded 69-bp oligonucleotide encoding an antisense ribozyme directed against mouse IDX-1 with incorporated 5'-EcoR I and 3'-Xba I restriction sites. The oligonucleotide sequences were as follows: sense strand, 5'-AATTCTTTCATCCACGGGAAAGGGAGCTGCTGATGAGTCCGTGAGGACGAAACGTTGGGCTCTTCT-3'; antisense strand, 5'-CTAGAGAAGAGCCCAACCGAGTTTCGTCCTCACGGACTCATCAGCAGCTCCCTTTCCCGTGGATGAAAG-3'. The double-stranded, annealed oligonucleotide was cloned into EcoRI/XbaI sites in the plasmid pUHD 10-3. The resulting Tet-ASRZIDX-1 plasmid sequence was verified by automated sequencing. The RIP-rtTA transgene was generated by excising a XhoI/AsnI fragment from the RIP-rtTA plasmid, and the Tet-ASRZ-IDX-1 transgene was generated by excising a XhoI/HindIII fragment from the Tet-ASRZ-IDX-1 plasmid. Transgenic mice were produced by microinjection of gel-purified transgene DNA into single-cell preimplantation FVB mouse embryos using standard methods $(30,31)$. For genotyping of transgenic mice, genomic DNA was isolated from tail biopsies (32), and transgenes were amplified by PCR. Islet expression for each of the RIP-rtTA and Tet-ASRZ-IDX-1 transgenes was confirmed by isolating pancreatic islets from genotyped transgenic mice, purifying islet RNA, and amplifying transgene products by RT-PCR (33). No PCR amplification of transgene products was observed from cDNA derived from pancreatic islets of nontransgenic control mice.

Doxycycline $(2 \mathrm{~g} / \mathrm{l}$ in $2.5 \%$ sucrose $)$ was administered in light-protected bottles in drinking water with sucrose supplementation to combat taste aversion from doxycycline (28). In selected studies, $15 \mathrm{mg}$ doxycycline or placebo 21-day release pellets (Innovative Research of America, Sarasota, Florida, USA) were implanted subcutaneously under isoflurane anesthesia. All studies were conducted in accordance with NIH guidelines for the care and use of laboratory animals and with approval from the Massachusetts General Hospital Institutional Animal Care and Use Committee.
Cell culture and transfections. HeLa and COS7 cells were obtained from American Type Culture Collection (Manassas, Virginia, USA). The clonal pancreatic $\beta$ cell lines MIN6 (34) and $\beta$ TC3 (35) were obtained from J. Miyazaki (Osaka University Medical School, Osaka, Japan) and S. Efrat (Sackler School of Medicine, Tel Aviv University, Ramat Aviv, Israel), respectively. MIN6 cells were cultured as described previously (34). All other cell lines were cultured in DMEM ( $4.5 \mathrm{~g} / \mathrm{l}$ glucose) supplemented with $10 \% \mathrm{FBS}, 100 \mathrm{U} / \mathrm{ml}$ of penicillin $\mathrm{G}$, and $100 \mathrm{mg} / \mathrm{ml}$ streptomycin sulfate (Life Technologies Inc., Gaithersburg, Maryland, USA). Tetracycline-free Tet System Approved FBS (CLONTECH Laboratories Inc., Palo Alto, California, USA) was used when it was available.

To generate double-stable rtTA/Tet-ASRZ-IDX-1 clonal cell lines, $\beta$ TC 3 cells were first transfected with a 10:1 ratio of the neomycin-resistance helper plasmid pSV2neo (CLONTECH) and the PCMV-rtTA plasmid pUHG 17-1, respectively, followed by selection with G418 (Calbiochem, La Jolla, California, USA) and amplification of individual rtTA- $\beta$ TC 3 cell clones. RtTA- $\beta$ TC 3 cell clones were tested for the ability to activate a transiently transfected heptamerized tet operator-luciferase reporter plasmid pUHC13-3 in a doxycycline-inducible manner. Functioning rtTA$\beta T C 3$ cell clones were then transfected with a 20:1 ratio of the hygromycin-resistance PTK-Hyg selection plasmid (CLONTECH) and the Tet-ASRZ-IDX-1 plasmid, respectively, followed by selection with G418 and hygromycin B (Boehringer Mannheim Corp., Indianapolis, Indiana, USA). Individual double-stable rtTA/Tet-ASRZ-IDX-1 clonal cell lines were amplified under G418 and hygromycin B selection. Doxycycline (Sigma Chemical Co., St. Louis, Missouri, USA) (1 $\mathrm{mg} / \mathrm{ml}$ stock solution in water), dexamethasone (Sigma Chemical Co.) (100 $\mu \mathrm{M}$ stock solution in $1 \%$ ethanol vehicle), or vehicle control solutions were administered to cell cultures as indicated.

Transfections were conducted with lipofectin or lipofectamine (Life Technologies Inc.), and luciferase and CAT assays were conducted as described $(25,33)$. Protein concentrations from cellular extracts were determined with the Bio-Rad Protein Assay (Bio-Rad Laboratories Inc., Hercules, California, USA). $P$ values were determined by Student's $t$ tests (Microsoft Excel; Microsoft Corp., Redmond, Washington, USA).

Western blots. Whole-cell extracts were separated by SDS-polyacrylamide gel electrophoresis and electroblotted on Immobilon-P membranes (Millipore Corp., Bedford, Massachusetts, USA). Total pancreatic protein extracts were prepared in an SDS lysis buffer (36) and protein concentrations were determined with a MicroBCA kit (Pierce Chemical Co., Rockford, Illinois, USA). Western blots were conducted as described elsewhere (26), with rabbit polyclonal anti-IDX-1 antiserum generated against the carboxy-terminal sequence of rat IDX-1 or rabbit polyclonal anti-Stat-3 (K-15) antiserum (Santa Cruz Biotechnology Inc., Santa Cruz, 
California, USA) and visualized with enhanced chemiluminescence using ECL Western blotting detection reagents (Amersham Life Sciences Inc., Arlington Heights, Illinois, USA). Data were scanned by computing densitometry (Molecular Dynamics, Sunnyvale, California, USA) and analyzed with ImageQuant software (Molecular Dynamics).

Glucose, insulin, and glycated hemoglobin measurements. Glucose levels were measured with a YSI 2300 STAT glucose analyzer (Yellow Springs Instrument Co. Inc., Yellow Springs, Ohio, USA). For glucose tolerance testing, mice were fasted for approximately 8 hours before intraperitoneal injection of $1.5 \mathrm{~g}$ glucose per kilogram of body weight. Plasma insulin levels were determined in duplicate with a rat insulin ELISA kit standardized with mouse insulin standards (Crystal Chem Inc., Chicago, Illinois, USA). Analyses of whole-blood samples for glycated hemoglobin levels were conducted with Glyc-Affin Ghb kits (Isolab Inc., Akron, Ohio, USA).

Northern blots. Whole pancreatic RNA was prepared with commercial nucleotide-binding columns (RNeasy; QIAGEN Inc., Valencia, California, USA), separated on formaldehyde agarose gels, and stained with ethidium bromide before Northern blotting conducted as described elsewhere (33). Blots were probed with a [ $\left.{ }^{32} \mathrm{P}\right]-$ radiolabeled probes directed against rat IDX-1 ( $5^{\prime}$-AGGTTACGGCACAATCCTGCTCCGGCTCTT- $3^{\prime}$ ) or $\beta$-actin (33).

\section{Results}

A model of inducible impairment of idx-1 expression. We designed a system of impairment of IDX-1 expression that does not disrupt the endogenous IDX-1 gene but rather alters the level of IDX-1 mRNA expression. To reduce the expression of IDX-1 mRNA we designed a combination antisense and ribozyme construct (Figure 1a). An endogenous GUC codon within the mouse IDX-1 mRNA sequence corresponding to Val 116 in the mouse IDX-1 protein defined the cleavage site of the ribozyme, designed in a hammerhead configuration employed by plant viruses (37, 38). The flanking antisense portion of the construct was complementary to 41 nucleotides of mouse IDX1 mRNA sequence with the ribozyme cleavage site located at a position $5^{\prime}$ of the IDX-1 homeodomain DNA-binding domain.

The reverse tetracycline transactivator (rtTA) system of inducible gene activation (29) was used to control the expression of the antisense ribozyme directed against IDX-1 (ASRZ-IDX-1). RtTA encodes a mutated tet repressor fused to the VP16 activation domain from the herpes simplex virus. In the absence of tetracycline analog such as doxycycline rtTA is unable to bind tet operator sequences. A target gene placed downstream of multimerized tet operator sequence remains transcriptionally inactive. Upon the addition of doxycycline, the conformation of rtTA changes so that it can bind to multimerized tet operator sequences and activate transcription of the target gene. The level of basal expression of the target gene in the absence of tetracycline analog administration defines the extent of leakiness inherent in the system. A twotransgene model was generated in which the target a

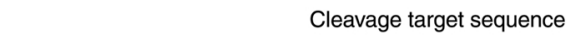

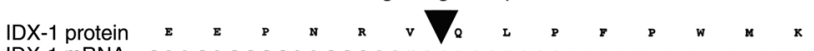

IDX-1 mRNA GAAGA GCC CAACCGCGUCCAGCUCCCUUUCCCGUGGA Antisense sequence 0 UCUCCGGGUVGUCGCA GUCGAGGGAAAGGGCACCUACUUD

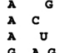

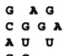

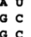
A C Hammerhead ribozyme

b
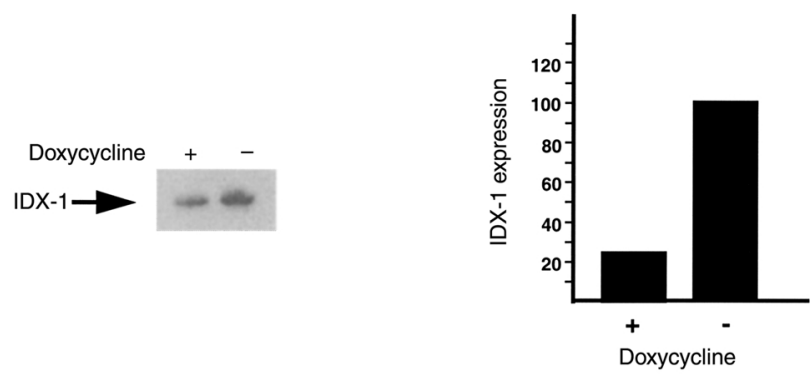

c

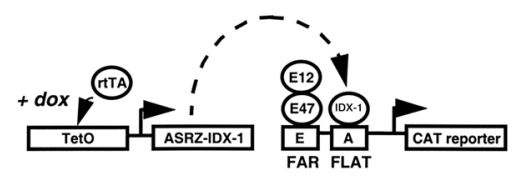

\section{Figure 1}

Inducible impairment of IDX-1 expression reduces insulin promoter activation. (a) Schematic model of the antisense ribozyme encoded within the Tet-ASRZ-IDX-1 transgene construct. The IDX-1 mRNA and corresponding protein sequences are aligned with the antisense ribozyme sequence. An arrowhead indicates the expected position of ribozyme cleavage. (b) Inducible impairment of ectopic expression of IDX-1 protein in HeLa cells. HeLa cells were transiently transfected (lipofectamine) with $0.5 \mu \mathrm{g}$ FarFlatCAT, 125 ng pCMV-IDX-1, 50 ng PCDNA3-E12, 50 ng pcDNA3-E47, $250 \mathrm{ng}$ Tet-ASRZ-IDX-1 transgene plasmid, and $4.0 \mu \mathrm{g}$ pCMV-rtTA (pUHG 17-1). As indicated (+), transfected cells were treated with $1 \mu \mathrm{g} / \mathrm{ml}$ doxycycline every 24 hours for a total of 48 hours before harvest and luciferase assay. Whole-cell extracts from transfected cells were analyzed by Western blotting with anti-IDX-1 antiserum. A corresponding autoradiograph is shown (left panels). By scanning densitometry of the Western blot, IDX-1 protein expression was reduced 4.3 -fold in response to doxycycline treatment (right panels). (c) Inducible impairment of IDX-1 expression reduces activation of glucose-responsive FarFlat enhancers within the rat insulin I promoter. Extracts from transfections of HeLa cells described for $\mathbf{b}$ were assayed for CAT activity. Data shown are the average of three transfections \pm SEM, each conducted in duplicate. Percent activation was determined by normalizing CAT activities to the activity of untreated transfected cells $\left({ }^{*} P \leq 0.01\right)$. 
gene is an antisense ribozyme targeted against IDX-1 (Tet-ASRZ-IDX-1). In the RIP-rtTA transgene, expression of rtTA was directed to pancreatic $\beta$ cells with the rat insulin II promoter (39). The model was designed so that the addition of a tetracycline analog such as doxycycline would increase expression of the antisense ribozyme, decrease levels of IDX-1 mRNA, and thereby reduce IDX-1 protein levels.

The function of the RIP-rtTA transgene construct was tested in the clonal pancreatic $\beta$ cell line $\beta$ TC3 (35) by cotransfection of the RIP-rtTA transgene construct and a tet-luciferase reporter plasmid pUHC 13-3 (27). Doxycycline increased the expression of the tetluciferase reporter 15-fold, indicating that the RIP-rtTA transgene construct was properly expressed in pancreatic $\beta$ cells and induced target gene expression in response to doxycycline.

Impairment of IDX-1 expression reduces activation of the insulin promoter. The function of the Tet-ASRZ-IDX-1 construct was assessed in COS7 cells in which the endogenous $I d x-1$ gene is not expressed. Cells were transiently transfected with an expression plasmid for rat IDX-1, a plasmid encoding rtTA regulated by the human CMV promoter (pUHG 17-1) (29), and the TetASRZ-IDX-1 plasmid. IDX-1 protein levels were assessed by Western blotting of whole-cell extracts. Doxycycline markedly attenuated IDX-1 expression indicating that induction of expression of the antisense ribozyme decreased IDX-1 levels.

We next reconstituted an experimental model system in HeLa cells in which the glucose-responsive elements Far (E) and Flat (A) of the rat insulin I promoter are known to be activated by the synergism between IDX-1 and the basic helix-loop-helix transcription factors $\mathrm{E} 12$ and $\mathrm{E} 47(15,24,40,41)$. HeLa cells were transiently transfected with expression plasmids encoding IDX-1, E12, E47, a FarFlat-CAT reporter plasmid, pCMV-rtTA (pUHG17-1) and Tet-ASRZ-IDX-1 plasmids. The administration of doxycycline reduced the expression of IDX-1 protein fourfold (Figure 1b). This degree of reduction in IDX-1 levels had significant functional consequences. Synergistic activation of the insulin promoter glucose-responsive elements FarFlat by coexpression of IDX-1 with the basic helix-loophelix transcription factors E12 and E47 $(15,24,40,41)$ was reduced in cells treated with doxycycline, indicating that IDX-1 levels can be rate-limiting for insulin gene activation (Figure 1c). These effects in transcriptional regulation in response to doxycycline were comparable in magnitude to the induction of luciferase a

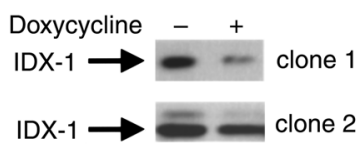

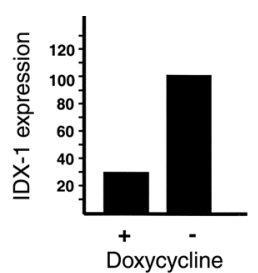

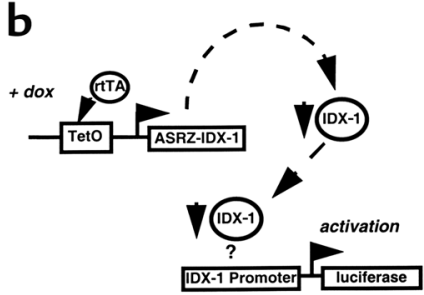

d

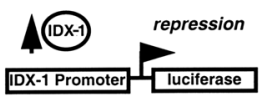

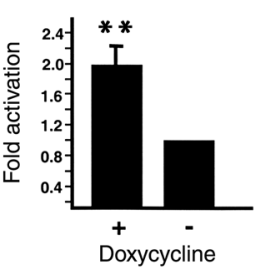

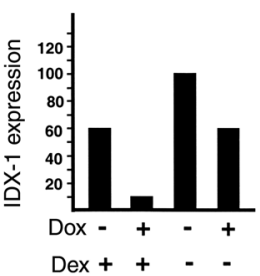

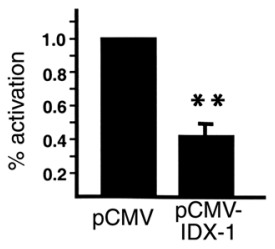

\section{Figure 2}

Impairment of IDX-1 expression in pancreatic $\beta$ cells activates the $I d x-1$ promoter. (a) Inducible impairment of IDX- 1 expression in clonal $\beta$ cells. Double-stable rtTA/Tet-ASRZ-IDX-1 clonal $\beta$ TC 3 cell lines were treated, as indicated $(+)$, with $1 \mu \mathrm{g} / \mathrm{ml} \mathrm{doxycycline} \mathrm{for} 72$ hours before harvest. Whole-cell extracts from two clonal cell lines were analyzed by Western blotting with anti-IDX-1 antiserum (left panels). Doxycycline-dependent reduction in IDX-1 expression ranged from $54 \%$ to $70 \%$ (clone 1 , right panel). (b) Idx-1 promoter activation in response to impairment of IDX-1 expression. Double-stable rtTA/Tet-ASRZ-IDX-1 clonal $\beta$ cells were transfected with $5 \mu \mathrm{g}-4.6 \mathrm{~kb}$ mouse $I d x-1$ promoter-luciferase reporter and treated, as indicated (+), with $1 \mu \mathrm{g} / \mathrm{ml} \mathrm{doxycycline} \mathrm{for} 48$ hours before harvest. Data shown are the average \pm SEM of three transfections in duplicate. Fold activation represents normalized luciferase activity relative to untreated transfected cells $\left({ }^{*} P \leq 0.01\right)$. (c) Dexamethasone enhances doxycycline-induced impairment of IDX-1 expression. Double-stable rtTA/Tet-ASRZIDX-1 clonal $\beta$ cell lines were treated with (+) or without $(-) 1 \mu \mathrm{g} / \mathrm{ml}$ doxycycline (Dox) for 72 hours before harvest and $100 \mathrm{nM}$ dexamethasone (Dex) for 48 hours before harvest. Western blots of whole-cell extracts from two clonal cell lines are shown (left panels). IDX-1 expression without doxycycline treatment was reduced $40-75 \%$ by dexamethasone, and the doxycycline-dependent reduction of IDX-1 expression was enhanced from $30 \%$ to $330 \%$ by dexamethasone (clone 3 , right panel). (d) Overexpression of IDX- 1 in $\beta$ cells decreases Idx-1 promoter activation. MIN6 cells were transiently transfected with $0.5 \mu \mathrm{g}$ pCMV-IDX-1 or pCMV empty expression vector, $3.5 \mu \mathrm{g}$ pBluescript, and $1 \mu \mathrm{g}-4.6 \mathrm{~kb}$ mouse $I d x-1$ promoter-luciferase reporter. Data shown are the average \pm SEM of three transfections in duplicate. Percent activation is normalized to the activity of cells transfected with $\mathrm{PCMV}$ alone $\left({ }^{*} P \leq 0.01\right)$. 
activity by doxycycline in HeLa cells cotransfected with pCMV-rtTA and tet-luciferase. The magnitude of inducible target gene regulation with the rtTA system often is reduced in transient transfection systems compared with stably transfected cells (29).

Activation of the IDX-1 promoter in response to impairment of IDX-1 expression in pancreatic $\beta$ cells. To analyze the efficacy of the impairment of IDX- 1 expression in pancreatic $\beta$ cells, we developed double-stable $\beta$ cell lines ( $\beta$ TC3) in which rtTA and Tet-ASRZ-IDX-1 were stably expressed under selection with G418 and hygromycin B. We analyzed multiple individual cell clones for efficiency of impairment of IDX-1 expression in response to doxycycline. For many of the double-stable clones, we observed $50-70 \%$ decreases in the expression of IDX-1 protein after 72 hours of exposure of the cells to doxycycline (Figure 2a). Doxycycline did not affect IDX-1 protein expression levels in stable $\beta$ cell lines in which rtTA alone was stably expressed. Further analysis of IDX-1 expression in response to doxycycline in selected double-stable clones over time revealed an interesting pattern. After a reduction in IDX-1 levels at 48-72 hours of doxycycline treatment, IDX-1 protein levels returned to the basal state after 92 hours of doxycycline exposure. We interpreted this pattern of IDX-1 expression as an indication that compensatory mechanisms exist within the pancreatic $\beta$ cell to maintain normal IDX-1 levels. Because IDX-1 is a critical regulator of pancreatic $\beta$ cell function, we reasoned that mechanisms likely exist in pancreatic $\beta$ cells to preserve cellular levels of IDX-1.

One potential mechanism to compensate for decreases in IDX-1 mRNA expression would be to increase IDX-1 mRNA production by activating the endogenous $I d x-1$ gene promoter to overcome the activity of the antisense ribozyme construct. We tested this possibility by transiently transfecting doublestable rtTA/Tet-ASRZ-IDX-1 $\beta$ cells with a $-4.6 \mathrm{~kb}$ mouse $I d x-1$ promoter-reporter construct. This segment of the mouse $I d x-1$ promoter contains the regulatory sequences necessary to faithfully impart the developmental and differentiated $\beta$ cell expression patterns of mouse IDX-1 (42). Treatment of the transfected double-stable rtTA/Tet-ASRZ-IDX-1 $\beta$ cells with doxycycline activated the $I d x-1$ promoter approximately twofold relative to untreated transfected cells (Figure 2b). On Northern blots within 20 hours of treatment of double-stable rtTA/Tet-ASRZ-IDX-1 $\beta$ cells with doxycycline, this compensatory mechanism was detected as an increase in IDX-1 mRNA levels of $25-60 \%$ compared with those of untreated control levels (normalized to $18 \mathrm{~S}$ rRNA and actin mRNA, respectively). The IDX-1 mRNA levels in this model system reflect the sum of the two processes of the reduction of IDX- 1 expression by the antisense ribozyme and the restoration of IDX-1 expression by activation of the $I d x-1$ promoter. These results indicated that the $I d x-1$ promoter is able to sense, either through direct or indi-
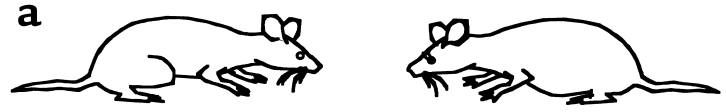

RIP-rtTA Tet-ASRZ-IDX-1

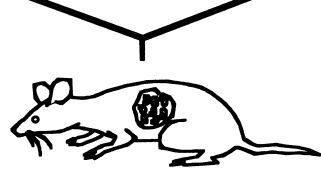

RIP-rtTA/Tet-ASRZ-IDX-1

b

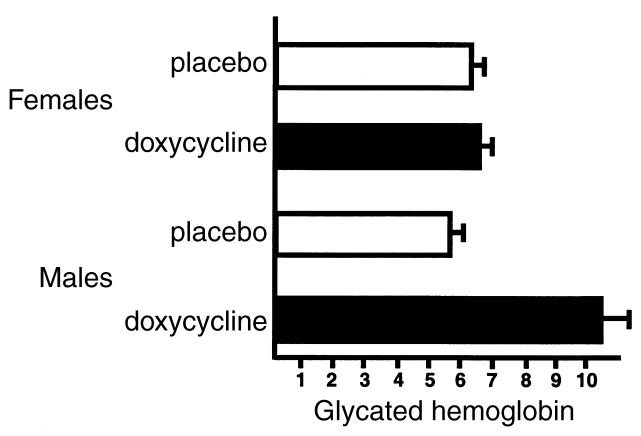

Figure 3

RIP-rtTA/Tet-ASRZ-IDX-1 double-transgenic mice exhibit inducible defects in glucose tolerance. (a) Schematic model of breeding strategy to generate RIP-rtTA/Tet-ASRZ-IDX-1 double-transgenic mice. Heterozygous or homozygous RIP-rtTA transgenic mice were mated with heterozygous or homozygous Tet-ASRZ-IDX-1 transgenic mice to generate double-transgenic progeny. (b) Inducible elevation of glycated hemoglobin levels in RIP-rtTA/Tet-ASRZ-IDX-1 double transgenic mice in a sexually dimorphic pattern. A litter of nine double-transgenic mice were administered subcutaneous 21-day slow-release placebo or doxycycline pellets as indicated for three consecutive cycles beginning at approximately 4 months of age. After 50 days of treatment, glycated hemoglobin levels were measured. Data shown reflect the average \pm range of measurements ( $n=2-3$ per sex and treatment group).

rect mechanisms, the levels of IDX-1 protein in the pancreatic $\beta$ cell. The $I d x-1$ promoter senses IDX-1 deficiency and is consequently activated thereby providing a mechanism to restore IDX-1 levels back to the normal ambient concentration.

To further evaluate the mechanism of $I d x-1$ promoter autoregulation, we selected double-stable rtTA/TetASRZ-IDX-1 $\beta$ cell clones in which minimal reductions of IDX-1 protein levels in response to doxycycline administration were observed. The addition of dexamethasone, an agent known to repress the activity of the $I d x-1$ promoter via an HNF $3 \beta$-mediated mechanism (23), to these double-stable clonal cell lines reduced basal IDX-1 protein levels by $40-75 \%$ as expected (Figure 2c). Interestingly, repression of $I d x-1$ promoter activation enhanced the ability of doxycycline to reduce IDX-1 protein expression by as much as $300 \%$ (Figure 2c). These findings suggested that the activity of the endogenous $I d x-1$ gene promoter limits the potency of the antisense ribozyme. The $I d x-1$ promoter likely senses both decreases and increases in IDX-1 expression levels. In transient transfections of 
$\mathbf{a}$

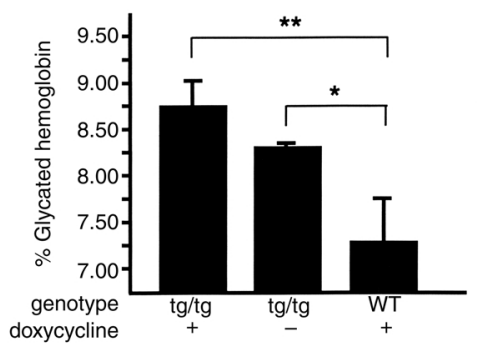

b
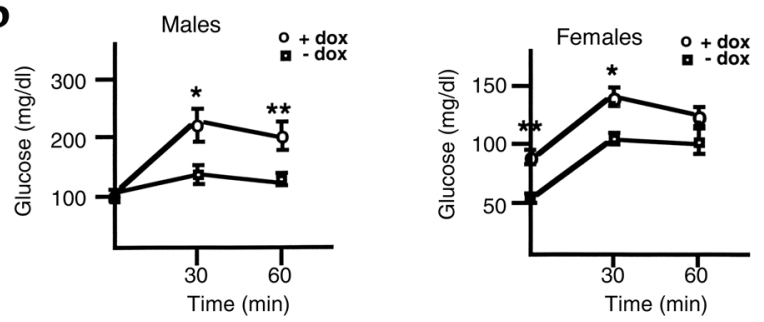

C

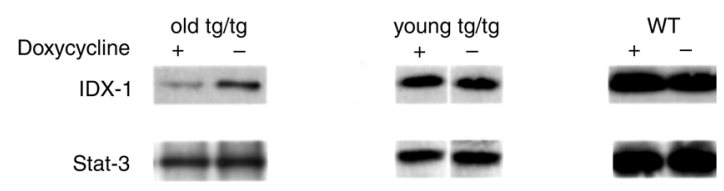

\section{Figure 4}

Induction of impairment of IDX-1 expression in older RIP-rtTA/TetASRZ-IDX-1 double-transgenic mice results in abnormal glucose tolerance. (a) Chronic doxycycline administration to older RIPrtTA/Tet-ASRZ-IDX-1 double-transgenic male mice increases glycated hemoglobin levels. Double transgenic ( $\mathrm{tg} / \mathrm{tg}$ ) or nontransgenic (WT) control male mice were treated with chronic oral doxycycline administration from weaning (doxycycline + ). Age-matched double-transgenic male mice that were not treated with doxycycline (doxycycline -) comprised a second control group. After the mice were 18 months of age or older, glycated hemoglobin levels were measured. Data shown are the mean \pm SEM of measurements from five to seven animals per group $\left({ }^{*} P \leq 0.05 ; * * \leq 0.01\right)$. (b) RIPrtTA/Tet-ASRZ-IDX-1 double-transgenic mice have inducible defects in glucose tolerance. Double-transgenic mice older than 18 months of age with (+ dox) or without (- dox) chronic oral doxycycline administration underwent intraperitoneal glucose tolerance testing. Data shown are the mean \pm SEM of measurements from four to six animals per treatment group for each $\operatorname{sex}\left({ }^{*} P<0.05 ;{ }^{*} P<0.01\right)$. (c) Inducible impairment of IDX-1 protein expression in older double-transgenic mice after chronic doxycycline treatment. Mouse pancreatic extracts were analyzed by Western blotting with anti-IDX-1 antiserum (upper panels) and anti-Stat-3 antiserum (lower panels) as a loading control. Data shown are from three separate Western blots. Extracts were derived from male double-transgenic mice older than 18 months of age (old $\mathrm{tg} / \mathrm{tg}$ ) with (+) or without (-) chronic oral doxycycline treatment and male double-transgenic (young $\mathrm{tg} / \mathrm{tg}$ ) or wild-type (WT) mice 3 months of age after a 3-week course of oral doxycycline $(+)$ or sucrose vehicle $(-)$ treatment.

the clonal pancreatic $\beta$ cell line MIN6, overexpression of an expression plasmid encoding exogenous IDX-1 suppressed activation of the $I d x-1$ promoter-reporter construct compared with cells transfected with the empty expression plasmid (Figure 2d). Thus IDX-1 levels appear to be regulated within a narrow range in pancreatic $\beta$ cells.
Inducible impairment of IDX-1 expression in transgenic mice. We generated two strains of transgenic mice, RIPrtTA and Tet-ASRZ-IDX-1 to avoid the integration of both transgenes in the same locus and minimize the levels of basal ribozyme expression in pancreatic $\beta$ cells (28). Three founder lines were bred and analyzed for the Tet-ASRZ-IDX-1 transgene, and two founder lines were bred and analyzed for the RIP-rtTA transgene. To generate a mouse model of inducible impairment of IDX-1 expression, we mated RIP-rtTA and Tet-ASRZIDX-1 mice and produced double-transgenic RIPrtTA/Tet-ASRZ-IDX-1 animals (Figure 3a). The double-transgenic mice developed normally in the absence or presence of doxycycline, unlike IDX-1 knockout mice that were born without a pancreas and died shortly after birth $(3,12)$. No differences were observed in body weight or pancreatic morphology between double-transgenic and nontransgenic littermate control mice. Oral administration of doxycycline in sucrose vehicle for 4 weeks to double-transgenic RIPrtTA/Tet-ASRZ-IDX-1 mice at 3-4 months of age did not impair glucose tolerance as assessed by intraperitoneal glucose tolerance testing.

As a paradigm for longer-term administration of doxycycline we subcutaneously implanted three consecutive cycles of 21-day slow-release doxycycline (15 $\mathrm{mg}$ ) or placebo pellets (Innovative Research of America) in each animal in a litter of double-transgenic RIPrtTA/Tet-ASRZ-IDX-1 mice that were generated from mating homozygous RIP-rtTA with homozygous TetASRZ-IDX-1 mice. After day 50 of treatment with doxycycline, we measured serum glycated hemoglobin levels in the 6-month-old animals. No significant differences in glycated hemoglobin levels were noted among the three female littermates treated with doxycycline and their two placebo-treated female littermates (Figure 3b). However, the two male littermates treated with doxycycline had substantially higher glycated hemoglobin levels than did their two placebo-treated male littermates (Figure 3b). These findings indicate a sexually dimorphic phenotypic pattern in this mouse model. Sexual dimorphism is a common finding in many rodent models of diabetes in which male mice have more marked abnormalities in glucose tolerance, including mouse models of defective insulin action (43). The metabolic phenotype in our double-transgenic mouse model was of low penetrance because six double-transgenic male mice from two subsequent litters did not develop elevated glycated hemoglobin levels in response to a similar course of subcutaneous doxycycline administration compared with five placebo-treated double-transgenic male littermate controls. Variable penetrance of diabetes has been observed in other mouse models $(43,44)$ and in humans with IDX-1 mutations (7) and likely reflects the variability in cosegregation of additional genes that confer susceptibility to or protection from the development of the disease.

Inducible diabetes mellitus with aging and IDX-1 deficiency. To obtain a phenotype of higher penetrance we treated 


\section{Table 1}

Diminished insulin/glucose ratios induced by impairment of IDX-1 expression

$\begin{array}{lccc} & 0 \min & 30 \min & 60 \min \\ \text { - Dox } & 18.4 \pm 6.5 & 34.4 \pm 12.4 & 31.0 \pm 11.0 \\ \text { + Dox } & 9.7 \pm 2.8 & 12.0 \pm 4.2^{\mathrm{A}} & 11.7 \pm 4.1^{\mathrm{A}}\end{array}$

Double transgenic RIP-rtTA/Tet-ASRZ-IDX-1 male mice older than 18 months of age with (+ Dox) or without (- Dox) chronic oral doxycycline administration underwent glucose-tolerance testing (Figure 4b). Insulin/glucose ratios \pm SEM at 0,30 , and 60 minutes after intraperitoneal glucose administration are shown ( $n=4$ per group; $\left.{ }^{A} P \leq 0.05\right)$.

double-transgenic RIP-rtTA/Tet-ASRZ-IDX-1 and nontransgenic control mice from the time of weaning with chronic oral doxycycline in a sucrose vehicle. At 3 months of age, no significant differences were observed in glucose tolerance between double-transgenic mice in the presence or absence of chronic oral doxycycline administration, as assessed by intraperitoneal glucose tolerance tests. At 8 months of age, no significant differences were found in glucose or glycated hemoglobin levels between double-transgenic mice in the presence or absence of chronic oral doxycycline administration.

However, after the mice were more than 18 months of age, significant differences were observed in glycated hemoglobin levels between double-transgenic RIP-rtTA/Tet-ASRZ-IDX-1 male mice and nontransgenic control mice after chronic doxycycline administration (Figure 4a). With chronic oral administration of doxycycline, the double transgenic male mice (Figure $4 \mathrm{a}, \mathrm{tg} / \mathrm{tg}$ ) had significantly higher glycated hemoglobin levels than did nontransgenic control male mice (Figure 4a, WT). Of note, double-transgenic male mice not treated with doxycycline had lower glycated hemoglobin levels than did age-matched double-transgenic male mice treated with chronic doxycycline, but they also had higher glycated hemoglobin levels than did nontransgenic controls. These data are consistent with a level of basal expression of the antisense ribozyme in the absence of tetracycline analog administration (leakiness in the transcriptional regulatory system) sufficient to induce abnormal glucose tolerance. To better characterize the phenotype in double-transgenic mice older than the age of 18 months induced by chronic oral doxycycline, we performed intraperitoneal glucose tolerance tests after 8hour fasts (Figure $4 \mathrm{~b}$ ). The chronic administration of doxycycline to the double-transgenic male mice resulted in significantly higher glucose excursions at 30 and 60 minutes relative to untreated double-transgenic age- and sex-matched mice. The impairment in glucose tolerance in males in this double-transgenic mouse model in response to doxycycline was accompanied by significant reductions in serum insulin/glucose ratios (Table 1). After treatment with doxycycline, variable impaired expression of IDX-1 protein was detected in pancreatic extracts derived from older but not younger double-transgenic male mice com- pared with untreated age- and sex-matched double transgenic controls (Figure 4c). With chronic doxycycline treatment the double-transgenic female mice did not have significant inducible elevations in glycated hemoglobin levels. Double-transgenic female mice had lower fasting and stimulated glucose levels than did male mice with small doxycycline-induced elevations of glucose levels (Figure 4b).

The metabolic dysfunction observed in these chronic doxycycline trials in older animals might have been attributable to the chronicity and duration of impairment of IDX-1 expression or to a difference in the sensitivity of older animals to the impairment of IDX-1 expression. To distinguish between these two possible explanations, we conducted a short-term trial of oral administration of doxycycline or sucrose vehicle in double-transgenic RIP-rtTA/Tet-ASRZ-IDX-1 male mice older than the age of 18 months (Figure 5a). After only 3 weeks of treatment, doxycycline-treated double-transgenic male mice had increased fasting glucose levels relative to their pretreatment baselines. In contrast, sucrose vehicle-treated double-transgenic mice showed no significant differences in fasting glu-
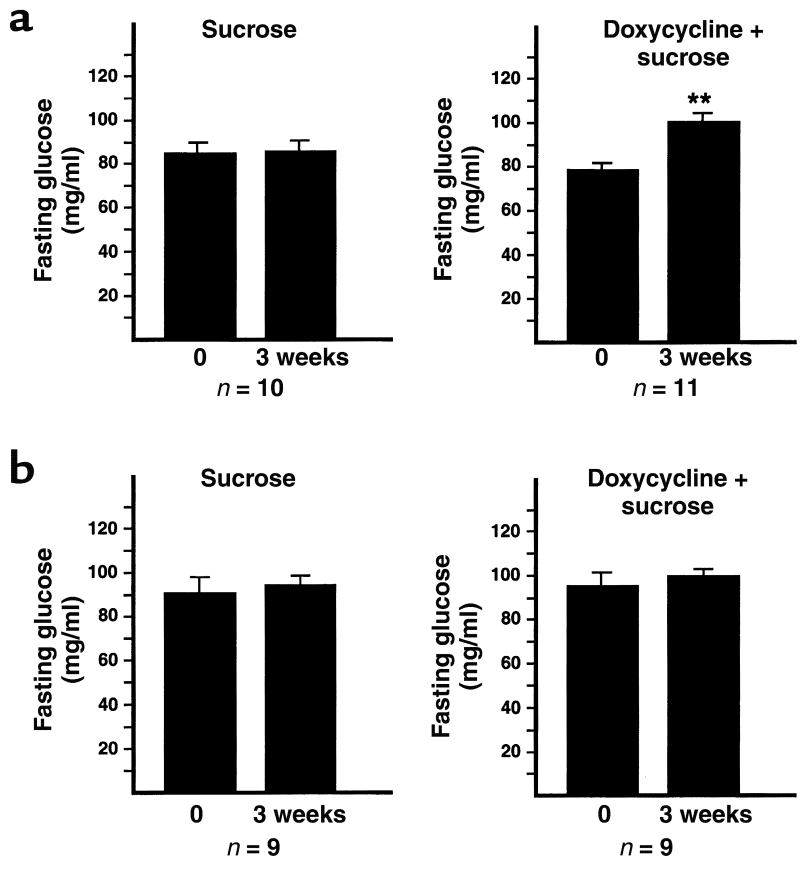

\section{Figure 5}

Short-term doxycycline administration induces fasting hyperglycemia in older but not younger RIP-rtTA/Tet-ASRZ-IDX-1 double-transgenic male mice. Baseline fasting blood glucose levels were measured from RIP-rtTA/Tet-ASRZ-IDX-1 double-transgenic male mice more than 18 months of age (a) or at 3 months of age (b) before a 3-week course of oral doxycycline (doxycycline + sucrose, right panels) or sucrose vehicle administration (sucrose, left panels). At the conclusion of the 3-week treatments, a second set of fasting blood glucose measurements was obtained. Data shown are the mean \pm SEM of measurements from 9 to 11 animals per treatment group as indicated $\left({ }^{*} P<0.01\right)$. Double-transgenic male mice served as their own pretreatment controls in this series of studies. 


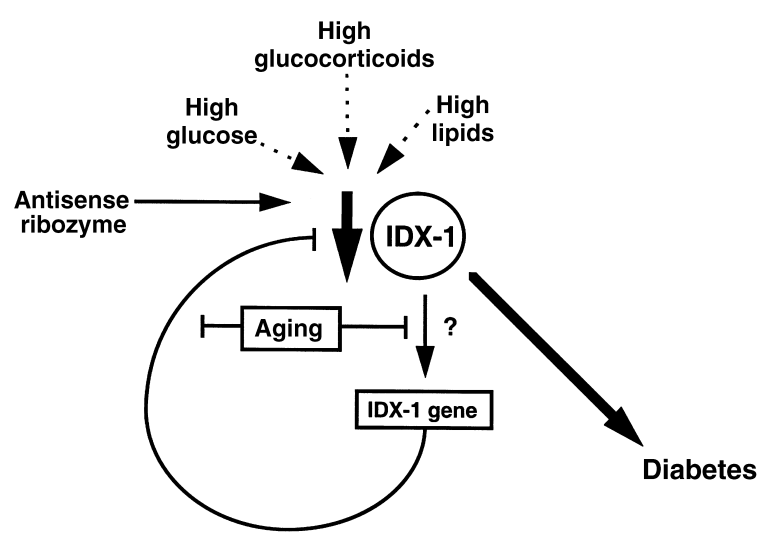

Figure 6

Schematic model whereby IDX-1 deficiency results in the development of diabetes mellitus. In the absence of mutations in the $I d x-1$ gene, pathophysiological processes that decrease IDX-1 levels may lead to defective glucose tolerance. Although our model used an antisense ribozyme to decrease IDX-1 expression, pathological conditions, such as elevated glucocorticoids, glucotoxicity from sustained hyperglycemia, or hyperlipidemia may reduce IDX-1 expression. In our model of inducible impairment of IDX-1 expression, older animals were more likely to develop metabolic abnormalities than were younger animals, likely owing to $\beta$ cell defects in sensing or response to IDX-1 deficiency.

cose levels before and after treatment. Double transgenic RIP-rtTA/Tet-ASRZ-IDX-1 female mice more than the age of 18 months had no significant increases in fasting glucose levels after 3 weeks of doxycycline treatment. Treatment of 3-month-old double-transgenic RIP-rtTA/Tet-ASRZ-IDX-1 male mice for 3 weeks with sucrose vehicle or doxycycline did not result in any significant differences in fasting glucose levels before and after treatment (Figure 5b). Doxycycline-dependent changes in pancreatic IDX-1 protein levels were not detected after 3 weeks of treatment of 3 -month-old double transgenic (Figure 4c, young $\mathrm{tg} / \mathrm{tg}$ ) male mice or nontransgenic male wild type (Figure 4c, WT) male control mice (Figure 4c). Nontransgenic wild-type 3-month-old male mice showed no significant differences in fasting glucose levels obtained after 3 weeks of treatment with sucrose-vehicle or doxycycline. These data suggest that older animals have increased sensitivity to the impairment of IDX-1 expression and demonstrate that short-term reduction of IDX-1 expression in older animals is sufficient to impair glucose tolerance.

\section{Discussion}

Deficiency of the pancreatic homeodomain transcription factor IDX-1 impairs glucose tolerance. In humans, IDX-1 appears to normally function within a limited concentration range because heterozygosity for mutations in the $I d x-1$ gene is sufficient to contribute to a metabolic phenotype (7-9). In other model systems, transcription factor dosage can provide a substantial impact. By altering expression levels of indi- vidual transcription factors, cell fate decisions and developmental programs are initiated or terminated $(45,46)$. Our studies of inducible IDX-1 deficiency suggest that even modest decrements in IDX-1 levels in differentiated pancreatic $\beta$ cells can have significant metabolic consequences.

In contrast to previous mouse models, the $I d x-1$ gene was not mutated or disrupted in our studies, thereby enabling normal regulatory mechanisms in the pancreatic $\beta$ cell to compensate for decreased IDX- 1 expression. A variety of possible regulatory responses are available within the $\beta$ cell to preserve IDX- 1 function in response to the reduction of IDX-1 mRNA levels including increasing rates of IDX-1 protein synthesis, IDX-1 protein stability, cytoplasmic to nuclear translocation of IDX-1 protein, expression of substitute transcriptional activators (including other homeodomain transcription factors), and $I d x-1$ gene transcription. We identified one such compensatory mechanism utilized by pancreatic $\beta$ cells in response to induction of expression of the antisense ribozyme targeted against IDX-1; namely, the activation of the $I d x-1$ promoter. The magnitude of this compensatory promoter activation was only approximately twofold, but within the expected range of differences in IDX-1 expression levels between humans heterozygous for $I d x-1$ mutations with a predisposition to the development of diabetes mellitus and humans without mutations. The implication of the observation that mutations in a single copy of the $I d x-1$ gene result in a metabolic phenotype is that compensatory mechanisms for IDX-1 deficiency rely heavily on two intact $I d x-1$ genes. Although alternate compensatory mechanisms that stabilize IDX-1 protein levels may still be operative in younger individuals, they are insufficient to prevent the development of defective glucose tolerance with aging in humans heterozygous for $I d x-1$ missense mutations.

We propose that IDX-1 levels regulate the activation of the $I d x-1$ promoter in an autoregulatory feedback loop in pancreatic $\beta$ cells. Our data suggest that the $I d x-1$ promoter is able to sense and respond to declining IDX-1 levels with increased transcriptional activation to restore levels of IDX-1 to the normal range. In the setting of excess IDX-1, as in our overexpression transfection studies (Figure $2 \mathrm{~d}$ ), the $I d x-1$ promoter responds with decreased transcriptional activation. Both of these proposed feedback mechanisms would serve to maintain IDX-1 protein levels in a tightly regulated range in the pancreatic $\beta$ cell. Mechanisms to alter $I d x-1$ promoter activation may be directly and/or indirectly mediated by the IDX-1 protein. Multiple AT-rich regions that could serve as potential direct binding sites for IDX-1 are present within the mouse and human $I d x-1$ promoters. The IDX-1 protein binds to oligonucleotides encoding one such AT-rich region within the human $I d x-1$ promoter that contributes to $\beta$ cell-specific expression (47). The expression of IDX-1 in NIH-3T3 cells activates an enhancer-reporter construct that incorporates this 
AT-rich region in synergy with HNF-3 $\beta$. On the basis of these studies, a positive feedback loop was proposed in which IDX-1 expression activates its own promoter (47). Our studies suggest that in the context of a pancreatic $\beta$ cell in which IDX-1 is normally expressed, overexpression of IDX-1 inhibits $I d x-1$ promoter activity. It is possible that IDX-1-mediated autoregulation may operate differently in distinct cellular or developmental contexts. Multiple enhancers are differentially used in pituitary development and in the autoregulation of expression of the homeoprotein Pit-1 (48), and it is conceivable that mechanisms of IDX-1 autoregulation will be of similar complexity. Insulin production declined in response to the impairment of IDX-1 expression, a finding that is consistent with the critical importance of IDX-1 in the regulation of the insulin gene. The magnitude of the inducible hyperglycemia may have been limited by extrapancreatic adjustments to decreased insulin production because increased insulin sensitivity has been reported in humans with $I d x-1$ mutations $(8,49)$.

The pattern of phenotypic sexual dimorphism observed in the RIP-rtTA/Tet-ASRZ-IDX-1 doubletransgenic mouse model is interesting and has not been reported in humans with $I d x-1$ mutations. A propensity for male mice to develop abnormalities in glucose tolerance more readily than female littermates is observed in other diabetes models (43), but the mechanisms responsible for such a pattern are unknown. Human females have lower fasting glucose levels (50) with reduced epinephrine, glucagon, and hepatic glucose production in response to insulin-induced hypoglycemia (51-53). Sex-related differences in neuroendocrine and metabolic responses to exercise also have been reported (54). The US Centers for Disease Control and Prevention report a steady increase in the prevalence of diabetes in Americans over the age of 65 from 1989 to 1996 , with a steeper rate of increase in males compared with females (55).

The convergence of IDX-1 deficiency, aging, and diabetes was somewhat surprising but underscores similar phenotypes among humans with IDX-1 mutations and mouse models. The severity of the mutations in the human $I d x-1$ gene correlate with the age of onset of metabolic dysfunction (7-9). In our mouse model, younger animals did not develop metabolic phenotypes in response to doxycycline administration, but after 18 months of age, male mice were highly susceptible to impairment of IDX- 1 expression. This phenotypic pattern suggests that mechanisms used by pancreatic $\beta$ cells to compensate for reductions in IDX-1 expression decline with aging (Figure 6). Of note, a mouse model of conditional $\beta$ cell-specific disruption of the IDX-1 gene using the cre/lox system did not develop diabetes until 4 months of age (11), but the age dependence of the phenotype may have been attributable to the time required for sufficient accumulation of cre expression in pancreatic $\beta$ cells to excise the targeted region of the $I d x-1$ gene. In contrast to our RIP-rtTA/Tet-ASRZ-IDX-1 transgenic mouse model, in the cre-lox model the endogenous $I d x-1$ gene is disrupted and would not be expected to respond with normal compensatory mechanisms.

Increasing insulin resistance has been identified as a major component in the increased risk of the development of type 2 diabetes with aging. However, we found no evidence of hyperinsulinemia in the agedependent inducible development of diabetes in our RIP-rtTA/Tet-ASRZ-IDX-1 double-transgenic mice. In fact, induction of IDX-1 deficiency resulted in diminished, not increased, insulin/glucose ratios. Agedependent reductions in pancreatic islet insulin production in rats have been reported (56). Our data imply that defects in pancreatic $\beta$ cell function, particularly in the ability to regulate IDX-1 expression, develop with aging. In other contexts, age-dependent changes in gene expression correlate with modifications in oxidative stress responses, caloric intake, mitotic regulation, and chromatin silencing (57-60).

The RIP-rtTA/Tet-ASRZ-IDX-1 double-transgenic model system of inducible IDX-1 deficiency has some intrinsic limitations. The extent of ASRZ-IDX-1 expression is directly regulated by rtTA expression, and thus indirectly limited by the activity of the rat insulin II promoter. Neogenesis, or the development of new pancreatic $\beta$ cells from precursor cells, is an active process in the endocrine pancreas (61). Newly developing pancreatic $\beta$ cells would not be susceptible to impairment of IDX-1 expression until insulin promoter activation was sufficient to promote rtTA expression, providing a potential mechanism to transiently bypass reduction of IDX-1 expression. In addition, the potency of the model system in IDX-1 inactivation is inherently limited by its design. Because IDX-1 is an important regulator of the activation of the insulin promoter, at some point a decrement in IDX-1 levels is likely to reduce rat insulin II promoter activity resulting in decreased rtTA expression, reducing ASRZ-IDX-1 expression and providing a limit to the extent of IDX-1 deficiency that can be induced. Furthermore, the higher glycated hemoglobin levels in double-transgenic mice in the absence of treatment with doxycycline implies a level of basal ASRZ-IDX-1 expression. This regulatory leak in our mouse model system limits the range of doxycyclineinducible differences in IDX-1 expression.

Although mutations in the $I d x-1$ gene are found in only $3-6 \%$ of individuals with type 2 diabetes $(8,9)$, our data suggest that abnormalities in glucose tolerance due to IDX-1 deficiency may occur in the larger population without IDX-1 mutations. Other investigators have identified a variety of insults that impair IDX-1 expression, including hyperglycemia, hyperlipidemia, and excess glucocorticoids (18-23). If aging diminishes the capacity of the pancreatic $\beta$ cell to restore impaired IDX-1 expression, older individuals are likely to be at greater risk for the subsequent development of diabetes. Thus, the development of therapeutic strategies to restore IDX-1 expression in set- 
tings of IDX-1 deficiency may be of particular importance for older adults. In this regard, activators of $I d x-1$ gene expression, such as glucagon-like peptide$1(36,62)$ and hedgehog signaling proteins $(26)$, warrant further investigation to assess their therapeutic potential in type 2 diabetes.

\section{Acknowledgments}

We thank H. Bujard for rtTA regulatory system plasmids, S. Efrat for $\beta$ TC3 cells, J. Miyazaki for MIN6 cells, J.L. Moss for FarFlat-CAT, and G.C. Weir for research support and insightful discussions. We appreciate the expert technical assistance of $\mathrm{H}$. Hermann and K. McManus. We thank D. Nathan and the hemoglobin A1C clinical laboratory at Massachusetts General Hospital for assistance with glycated hemoglobin assays. We are indebted to the members of the Laboratory of Molecular Endocrinology for valuable discussions. We also thank T. Budde for assistance in the preparation of this manuscript. These studies were supported by grants DK02476 and DK58783 (M.K. Thomas) and DK30457 and DK30834 (J.F. Habener) from the NIH. M.K. Thomas is the recipient of a Howard Hughes Medical Institute Postdoctoral Fellowship for Physicians. J.F. Habener is an Investigator with the Howard Hughes Medical Institute.

1. Ohlsson, H., Karlsson, K., and Edlund, T. 1993. IPF1, a homeodomaincontaining transactivator of the insulin gene. EMBO J. 12:4251-4259.

2. Leonard, J., et al. 1993. Characterization of somatostatin transactivating factor-1, a novel homeobox factor that stimulates somatostatin expression in pancreatic islet cells. Mol. Endocrinol. 7:1275-1283.

3. Offield, M.F., et al. 1996. PDX-1 is required for pancreatic outgrowth and differentiation of the rostral duodenum. Development. 122:983-995.

4. Marshak, S., Totary, H., Cerasi, E., and Melloul, D. 1996. Purification of the beta-cell glucose-sensitive factor that transactivates the insulin gene differentially in normal and transformed islet cells. Proc. Natl. Acad. Sci. USA. 93:15057-15062.

5. Stoffers, D.A., Thomas, M.K., and Habener, J.F. 1997. Homeodomain protein IDX-1: a master regulator of pancreas development and insulin gene expression. Trends Endocrinol. Metab. 8:145-151.

6. Edlund, H. 1998. Transcribing pancreas. Diabetes. 47:1817-1823.

7. Stoffers, D.A., Ferrer, J., Clarke, W.L., and Habener, J.F. 1997. Earlyonset type-II diabetes mellitus (MODY4) linked to IPF-1. Nat. Genet. 17:138-139.

8. Hani, E.H., et al. 1999. Defective mutations in the insulin promoter factor-1 (IPF-1) gene in late-onset type 2 diabetes mellitus. J. Clin. Invest. 104:R41-R48.

9. Macfarlane, W.M., et al. 1999. Missense mutations in the insulin promoter factor- 1 gene predispose to type 2 diabetes. J. Clin. Invest. 104:R33-R39.

10. Dutta, S., Bonner-Weir, S., Montminy, M., and Wright, C. 1998. Regulatory factor linked to late-onset diabetes? Nature. 392:560.

11. Ahlgren, U., Jonsson, J., Jonsson, L., Simu, K., and Edlund, H. 1998. $\beta$ cell-specific inactivation of the mouse $I P f 1 / P d x 1$ gene results in loss of the $\beta$-cell phenotype and maturity onset diabetes. Genes Dev. 12:1763-1768.

12. Jonsson, J., Carlsson, L., Edlund, T., and Edlund, H. 1994. Insulin-promoter-factor 1 is required for pancreas development in mice. Nature. 371:606-609.

13. Stoffers, D.A., Zinkin, N.T., Stanojevic, V., Clarke, W.L., and Habener, J.F. 1997. Pancreatic agenesis attributable to a single nucleotide deletion in the human IPF1 coding region. Nat Genet. 15:106-110.

14. German, M.S., Moss, L.G., Wang, J., and Rutter, W.J. 1992. The insulin and islet amyloid polypeptide genes contain similar cell-specific promoter elements that bind identical beta-cell nuclear complexes. Mol. Cell. Biol. 12:1777-1788.

15. Peers, B., Leonard, J., Sharma, S., Teitelman, G., and Montminy, M.R. 1994. Insulin expression in pancreatic islet cells relies on cooperative interactions between the helix loop helix factor E47 and the homeobox factor STF-1. Mol. Endocrinol. 8:1798-1806.

16. Waeber, G., Thompson, N., Nicod, P., and Bonny, C. 1996. Transcriptional activation of the GLUT2 gene by the IPF-1/STF-1/IDX-1 homeobox factor. Mol. Endocrinol. 10:1327-1334.

17. Watada, H., et al. 1996. PDX-1 induces insulin and glucokinase gene expressions in $\alpha \mathrm{TC} 1$ clone 6 cells in the presence of betacellulin. Diabetes. 45:1826-1831.

18. Olson, L.K., et al. 1995. Reduction of insulin gene transcription in HITT15 $\beta$ cells chronically exposed to a supraphysiologic glucose concentration is associated with loss of STF-1 transcription factor expression. Proc. Natl. Acad. Sci. USA. 92:9127-9131.

19. Sharma, A., Olson, L.K., Robertson, R.P., and Stein, R. 1995. The reduction of insulin gene transcription in HIT-T15 $\beta$ cells chronically exposed to high glucose concentration is associated with the loss of RIPE3b1 and STF-1 transcription factor expression. Mol. Endocrinol. 9:1127-1134

20. Zangen, D.H., et al. 1997. Reduced insulin, GLUT2, and IDX-1 in $\beta$ cells after partial pancreatectomy. Diabetes. 46:258-264.

21. Seufert, J., Weir, G.C., and Habener, J.F. 1998. Differential expression of the insulin gene transcriptional repressor CCAAT/enhancer-binding protein beta and transactivator islet duodenum homeobox-1 in rat pancreatic beta cells during the development of diabetes mellitus. $J$. Clin. Invest. 101:2528-2539.

22. Gremlich, S., Bonny, C., Waeber, G., and Thorens, B. 1997. Fatty acids decrease IDX-1 expression in rat pancreatic islets and reduce GLUT2, glucokinase, insulin, and somatostatin levels. J. Biol. Chem. 272:30261-30269.

23. Sharma, S., et al. 1997. Hormonal regulation of an islet-specific enhancer in the pancreatic homeobox gene STF-1. Mol. Cell. Biol. 17:2598-2604.

24. Stoffers, D.A., Stanojevic, V., and Habener, J. 1998. Insulin promoter factor- 1 gene mutation linked to early-onset type 2 diabetes mellitus directs expression of a dominant negative isoprotein. J. Clin. Invest. 102:232-241.

25. Thomas, M.K., Yao, K.M., Tenser, M.S., Wong, G.G., and Habener, J.F. 1999. Bridge-1, a novel PDZ-domain coactivator of E2A-mediated regulation of insulin gene transcription. Mol. Cell. Biol. 19:8492-8504.

26. Thomas, M.K., Lee, J.H., Rastalsky, N., and Habener, J.F. 2001. Hedgehog signaling regulation of homeodomain protein IDX-1 expression in pancreatic $\beta$ cells. Endocrinology. 142:1033-1040.

27. Gossen, M., and Bujard, H. 1992. Tight control of gene expression in mammalian cells by tetracycline-responsive promoters. Proc. Natl. Acad. Sci. USA. 89:5547-5551.

28. Kistner, A., et al. 1996. Doxycycline-mediated quantitative and tissuespecific control of gene expression in transgenic mice. Proc. Natl. Acad. Sci. USA. 93:10933-10938.

29. Gossen, M., et al. 1995. Transcriptional regulation by tetracyclines in mammalian cells. Science. 268:1766-1769.

30. Gordon, J., and Ruddle, F.H. 1983. Gene transfer into mouse embryos: production of transgenic mice by pronuclear injection. Methods Enzymol. 89:411-433.

31. Hogan, B., Constantini, F., and Lacy, E. 1986. Manipulating the mouse embryo: a laboratory manual. Cold Spring Harbor Laboratory Press. New York, New York, USA. 487 pp.

32. Laird, P.W., et al. 1991. Simplified mammalian DNA isolation procedure. Nucleic Acids Res. 19:4293.

33. Thomas, M.K., Rastalsky, N., Lee, J., and Habener, J.F. 2000. Hedgehog signaling regulates insulin production by pancreatic $\beta$-cells. Diabetes. 49:2039-2047.

34. Miyazaki, J., et al. 1990. Establishment of a pancreatic beta cell line that retains glucose-inducible insulin secretion: special reference to expression of glucose transporter isoforms. Endocrinology. 127:126-132.

35. Efrat, S., Surana, M., and Fleischer, N. 1991. Glucose induces insulin gene transcription in a murine pancreatic $\beta$-cell line. J. Biol. Chem. 266:11141-11143.

36. Stoffers, D.A., et al. 2000. Insulinotropic glucagon-like peptide 1 agonists stimulate expression of homeodomain protein IDX-1 and increase islet size in mouse pancreas. Diabetes. 49:741-748.

37. Haseloff, J., and Gerlach, W.L. 1988. Simple RNA enzymes with new and highly specific endoribonuclease activities. Nature. 334:585-591.

38. Scanlon, K.J., et al. 1995. Oligonucleotide-mediated modulation of mammalian gene expression. FASEB J. 9:1288-1296.

39. Hanahan, D. 1985. Heritable formation of pancreatic $\beta$-cell tumors in transgenic mice expressing recombinant insulin/simian virus 40 oncogenes. Nature. 315:115-122.

40. German, M.S., Wang, J., Chadwick, R.B., and Rutter, W.J. 1992. Synergistic activation of the insulin gene by a LIM-homeodomain protein and a basic helix-loop-helix protein: building a functional insulin minienhancer complex. Genes Dev. 6:2165-2176.

41. Ohneda, K., Mirmira, R.G., Wang, J., Johnson, J.D., and German, M.S. 2000. The homeodomain of PDX-1 mediates multiple protein-protein 
interactions in the formation of a transcriptional activation complex on the insulin promoter. Mol. Cell. Biol. 20:900-911.

42. Stoffers, D.A., Heller, R.S., Miller, C.P., and Habener, J.F. 1999. Developmental expression of the homeodomain protein IDX-1 in mice transgenic for an IDX-1 promoter/lacZ transcriptional reporter. Endocrinology. 140:5374-5381.

43. Brüning, J.C., et al. 1997. Development of a novel polygenic model of NIDDM in mice heterozygous for IR and IRS-1 null alleles. Cell. 88:561-572

44. Kido, Y., et al. 2000. Tissue-specific insulin resistance in mice with mutations in the insulin receptor, IRS-1, and IRS-2. J. Clin. Invest. 105:199-205.

45. Greer, J.M., Puetz, J., Thomas, K.R., and Capecchi, M.R. 2000. Maintenance of functional equivalence during paralogous Hox gene evolution. Nature. 403:661-665.

46. DeKoter, R.P., and Singh, H. 2000. Regulation of B lymphocyte and macrophage development by graded expression of PU.1. Science. 288:1439-1441.

47. Marshak, S., et al. 2000. Functional conservation of regulatory elements in the pdx-1 gene: PDX-1 and hepatocyte nuclear factor $3 \beta$ transcription factors mediate $\beta$-cell-specific expression. Mol. Cell. Biol. 20:7583-7590.

48. DiMattia, G.E., et al. 1997. The PIT-1 gene is regulated by distinct early and late pituitary-specific enhancers. Dev. Biol. 182:180-90.

49. Clocquet, A.R., et al. 2000. Impaired insulin secretion and increased insulin sensitivity in familial maturity-onset diabetes of the young 4 (insulin promoter factor 1 gene). Diabetes. 49:1856-1864.

50. Merimee, T.J., and Tyson, J.E. 1974. Stabilization of plasma glucose during fasting. N. Engl. J. Med. 291:1275-1278.

51. Amiel, S.A., Maran, A., Powrie, J.K., Umpleby, A.M., and Macdonald, I.A. 1993. Gender differences in counterregulation to hypoglycemia. Diabetologia. 36:460-464.

52. Davis, S.N., Cherrington, A.D., Goldstein, R.E., Jacobs, J., and Price, L.
1993. Effects of insulin on the counterregulatory response to equivalent hypoglycemia in normal females. Am. J. Physiol. 265:E680-E689.

53. Fanelli, C., et al. 1994. Relative roles of insulin and hypoglycaemia on induction of neuroendocrine responses to, symptoms of, and deterioration of cognitive function in hypoglycaemia in male and female humans. Diabetologia. 37:797-807.

54. Davis, S.N., Galassetti, P., Wasserman, D.H., and Tate, D. 2000. Effects of gender on neuroendocrine and metabolic counterregulatory responses to exercise in normal man. J. Clin. Endocrinol. Metab. 85:224-230.

55. National Center for Chronic Disease Prevention and Health Promotion. 1999. Diabetes public health resource, statistics diabetes surveillance. Tables 2.9-2.10. http://www.cdc.gov/diabetes/statistics/survl99/ chap2/contents.htm.

56. Perfetti, R., Rafizadeh, C.M., Liotta, A.S., and Egan, J.M. 1995. Agedependent reduction in insulin secretion and insulin mRNA in isolated islets from rats. Am. J. Physiol. 269:E983-E990.

57. Lee, C.-K., Klopp, R.G., Weindruch, R., and Prolla, T.A. 1999. Gene expression profile of aging and its retardation by caloric restriction. Science. 285:1390-1393.

58. Guarente, L. 2000. Sir2 links chromatin silencing, metabolism, and aging. Genes Dev. 14:1021-1026.

59. Ly, D.H., Lockhart, D.J., Lerner, R.A., and Schultz, P.G. 2000. Mitotic misregulation and human aging. Science. 287:2486-2491.

60. Zou, S., Meadows, S., Sharp, L., Jan, L.Y., and Jan, Y.N. 2000. Genomewide study of aging and oxidative stress response in Drosophila melanogaster. Proc. Natl. Acad. Sci. USA. 97:13726-13731.

61. Finegood, D.T., Scaglia, L., and Bonner-Weir, S. 1995. Dynamics of $\beta$ cell mass in the growing rat pancreas: estimation with a simple mathematical model. Diabetes. 44:249-256.

62. Wang, X., et al. 1999. Glucagon-like peptide-1 regulates the beta cell transcription factor, PDX-1, in insulinoma cells. Endocrinology. 140:4904-4907. 